\title{
Avaliação do nível de satisfação de usuários de aparelhos de amplificação sonora individuais dispensados pelo Sistema Único de Saúde
}

\author{
Satisfaction evaluation in users of hearing aid dispensed by the \\ Unified Health System
}

\author{
Fabiana Danieli ${ }^{1}$, Eliane Aparecida Techi Castiquini ${ }^{2}$, Ticiana Cristina de Freitas Zambonatto ${ }^{2}$, \\ Maria Cecília Bevilacqua ${ }^{3}$
}

\begin{abstract}
RESUMO
Objetivo: Adaptar culturalmente o questionário Satisfaction with Amplification in Daily Life (SADL), versão em Português Brasileiro, para administrar em usuários de aparelhos de amplificação sonora individuais (AASIs) dispensados pelo Sistema Único de Saúde (SUS). Métodos: Participaram da pesquisa 19 sujeitos usuários de AASIs dispensados pelo Sistema Único de Saúde, por no mínimo três semanas, com idade igual ou superior a 60 anos, que apresentavam perda auditiva pós-lingual, sendo $63 \%$ do gênero masculino e $37 \%$ do gênero feminino, com média de idade de 73 anos e média de tempo de uso das próteses auditivas de cinco meses. Os sujeitos responderam ao questionário SADL e a uma Escala de Satisfação de item único, além de alguns itens adicionais. Foi realizada a análise da consistência interna do SADL, a análise descritiva dos resultados, para caracterizar as respostas dos sujeitos, a comparação dos resultados com os dados normativos propostos pelos autores e uma investigação do relacionamento entre o SADL e a escala de satisfação de item único. Resultados: Os resultados obtidos para a pontuação global evidenciaram que os sujeitos, em média, encontravam-se satisfeitos com seus AASIs. O mesmo ocorreu para todas as subescalas do SADL, sendo que a subescala Imagem Pessoal apresentou o maior número de pessoas muito satisfeitas. Os resultados obtidos foram equivalentes aos encontrados pelos autores e o SADL mostrou ser um questionário com boa consistência interna $(0,71)$. Houve íntima relação entre a escala de satisfação de item único e a pontuação global do SADL. Para as duas medidas de satisfação, a maioria dos sujeitos demonstrou estar "satisfeito" com seus AASIs e ambas as escalas apresentaram correlação alta e significativa $(0,935)$. Conclusão: Em média, os sujeitos estavam satisfeitos com seus AASIs dispensados pelo SUS. O questionário SADL, versão em Português Brasileiro, mostrou-se um instrumento eficaz para avaliar o nível de satisfação dos usuários de AASIs dispensados pelo SUS avaliados neste estudo. Novas pesquisas são necessárias para complementar estes achados.
\end{abstract}

Descritores: Auxiliares de audição; Perda auditiva; Satisfação dos consumidores; Saúde pública; Sistema Único de Saúde

\section{INTRODUÇÃO}

A audição é um dos sentidos fundamentais à vida e de-

Trabalho realizado na Divisão de Saúde Auditiva do Hospital de Reabilitação de Anomalias Craniofaciais, Universidade de São Paulo - USP - Bauru (SP), Brasil.

Os autores declaram a inexistência de conflitos de interesse.

(1) Programa de Pós-Graduação Interunidades em Bioengenharia EESC/ FMRP/IQSC - USP - São Carlos (SP), Brasil.

(2) Hospital de Reabilitação de Anomalias Craniofaciais, Universidade de São Paulo - USP - Bauru (SP), Brasil.

(3) Programa de Implante Coclear do Centro de Pesquisas Audiológicas do Hospital de Reabilitação de Anomalias Craniofaciais, Universidade de São Paulo - USP - Bauru (SP), Brasil.

Endereço para correspondência: Fabiana Danieli. Centro de Pesquisas Audiológicas (CPA). R. Octávio Pinheiro Brisola, 9/75, Bauru (SP), Brasil, CEP: 17012-901.E-mail: fabianadanieli@ hotmail.com

Recebido em: 5/6/2009; Aceito em: 28/9/2010 sempenha um importante papel na sociedade, pois é base do desenvolvimento da comunicação humana. Um indivíduo com incapacidade auditiva pode sofrer sérios danos em sua vida social, psicológica e profissional. Sentimentos como insegurança, medo, depressão, isolamento e tensão podem surgir e agravar-se devido à falta de atenção adequada dispensada ao indivíduo com deficiência auditiva.

A (re)habilitação auditiva pode ser definida como um processo de resolução de problemas, com o objetivo de minimizar a dificuldade (limitação de atividade) e a desvantagem (restrição de participação) de um indivíduo com uma deficiência de audição ${ }^{(1)}$.

A seleção de um dispositivo de amplificação é um componente crucial nesse processo, e constitui-se no principal recurso utilizado para tentar compensar as desvantagens da deficiência auditiva, quando não há tratamento médico ou cirúrgico pos- 
sível. O aparelho de amplificação sonora individual (AASI) é um dispositivo eletrônico, miniaturizado, que tem a função de amplificar os sons de modo a permitir a estimulação da audição residual, melhorando, assim, a capacidade auditiva do indivíduo com deficiência auditiva.

Embora os AASIs tenham evoluído muito nesses últimos anos em termos de qualidade e versatilidade, o sucesso da amplificação continua sendo um desafio para os profissionais e as altas taxas de abandono do uso da prótese auditiva um problema para os serviços de saúde.

Vários fatores acústicos e não-acústicos influenciam no sucesso e no fracasso individual do uso da amplificação ${ }^{(2)}$. O sucesso da amplificação depende de inúmeros fatores, como grau da perda auditiva, índices de reconhecimento de fala e expectativas e motivação do paciente, entre outros ${ }^{(3)}$.

Dessa forma, diversas medidas foram desenvolvidas com o objetivo de demonstrar as mudanças clínicas observadas para variáveis particulares de intervenção, promovidas no mundo real onde o serviço é fornecido. Nos setores de (re)habilitação auditiva, as medidas de resultado tem emergido como um método efetivo para determinar se uma intervenção específica, como o AASI, está promovendo resultados positivos para seus usuários ${ }^{(4)}$.

As medidas de resultado utilizadas pelos serviços de fornecimento de AASI, tanto no setor público quanto no setor privado, geralmente envolvem o uso de questionários auto-administrados, padronizados, centrados no paciente e denominados questionários de auto-avaliação ${ }^{(5)}$.

Os questionários de auto-avaliação possibilitam uma avaliação do impacto da perda auditiva na vida diária do indivíduo, o planejamento e a execução de estratégias de reabilitação voltadas às necessidades da pessoa com perda auditiva, e ainda, podem ser usados para documentar o mérito do programa de tratamento, bem como para pontuar áreas que necessitam de melhorias, aperfeiçoando e demonstrando a efetividade dos serviços de saúde ${ }^{(4)}$.

A opinião do paciente sobre a qualidade do tratamento (dispositivo, aconselhamento associado e outros serviços) é considerada como um indicador fortemente significativo de excelência do tratamento ${ }^{(6)}$.

No Brasil, o Ministério da Saúde instituiu em 2004, por meio da Portaria GM/MS n ${ }^{\circ} .2 .073^{(7)}$, a Política Nacional de Atenção à Saúde Auditiva. A criação desta portaria surgiu com a necessidade de aprimoramento e efetividade dos procedimentos de concessão de AASIs, promovendo uma organização das ações direcionadas à atenção básica e estruturando os serviços de média e alta complexidade.

A constituição destas redes possibilitou um grande avanço no aprimoramento das ações de saúde auditiva do Sistema Único de Saúde (SUS), na medida em que propôs a organização de uma rede hierarquizada, regionalizada e integrada aos diversos serviços de saúde, contemplando ações de promoção e proteção, diagnóstico, seleção, fornecimento de aparelho auditivo e reabilitação ${ }^{(8)}$.

Pacientes com problemas de audição entram nos serviços de saúde auditiva com o objetivo de melhorar sua função auditiva e consequentemente seu desempenho comunicativo. Quando o tratamento é fornecido, como a concessão do aparelho auditivo ou o serviço de reabilitação, ou ambos, a efetividade do tratamento precisa ser avaliada ${ }^{(9)}$.

$\mathrm{Na}$ literatura referente à Audiologia, muito se tem estudado em relação ao benefício e à satisfação do paciente em relação ao uso do AASI, como forma de avaliar os resultados da intervenção ${ }^{(10)}$.

Baseado em uma extensa investigação com usuários de AASI, um estudo ${ }^{(2)}$ investigou os fatores considerados pelos consumidores como sendo críticos para a satisfação com a amplificação. Tal estudo revelou que a melhora na habilidade auditiva, qualidade sonora apropriada, confiança no instrumento, utilização em múltiplos ambientes auditivos, manutenção, e adaptaçãolconforto foram os mais importantes fatores que influenciaram a satisfação dos consumidores. Isto sugere que a quantificação de resultados da adaptação na perspectiva do cliente pode ser definida utilizando medidas mais abrangentes do que o uso e o desempenho com o aparelho auditivo.

Dessa forma, "embora um cliente possa demonstrar beneficio quantificável com seu AASI, como uma melhora na habilidade de compreender a fala de outras pessoas, isto não assegura que o cliente esteja totalmente satisfeito com o serviço global recebido e também não garante que o cliente considere a prótese auditiva vantajosa" ${ }^{(11)}$.

Como resultado da intervenção com o AASI, satisfação é um conceito complexo que não reflete somente a percepção do paciente com relação ao beneficio do próprio dispositivo (incluindo desempenho, conforto, aparência, etc), mas também pode referir-se a questões externas ao tratamento, como a qualidade do serviço, o ambiente de atenção, o custo, o valor percebido, e a popularidade do fornecedor ${ }^{(12)}$.

Ao identificar os fatores que contribuem para a satisfação e ao tentar prover tais atributos aos processos envolvidos, tem-se o potencial de obter um resultado mais efetivo nos serviços de saúde ${ }^{(13)}$.

O questionário Satisfaction with Amplification in Daily Life (SADL $)^{(4)}$ foi elaborado com o intuito de quantificar a satisfação global com o AASI e de gerar um perfil de satisfação em cada um dos quatro maiores domínios da satisfação.

Foi desenvolvido em vários estágios. Inicialmente, os elementos mais importantes para satisfação pela maioria das pessoas foram relatados por meio de entrevistas estruturadas. Então, itens experimentais de satisfação foram criados para cada área de importância e um questionário de satisfação contendo 25 itens foi gerado e distribuído para outro grupo de usuários de AASI. Resultados foram obtidos de 257 indivíduos, e estes resultados foram analisados para gerar um questionário final. $\mathrm{O}$ questionário apresentou alta confiabilidade na correlação teste-reteste $(0,81)$ e sua versão final se mostrou relativamente curta, contendo 15 itens divididos em quatro subescalas, descritas a seguir:

- Efeitos positivos (questões 1, 3, 5, 6, 9 e 10): seis itens referentes ao benefício acústico e psicológico;

- Serviço e custo (questões 12, 14 e 15): três itens referentes à competência profissional, preço do produto e número de consertos;

- Fatores negativos (questões 2, 7 e 11): três itens referentes à amplificação de ruído ambiental, presença de microfonia e uso do telefone; 
- Imagem pessoal (questões 4, 8 e 13): três itens referentes à estética e ao estigma do uso do AASI.

O SADL foi validado ${ }^{(14)}$ em uma amostra de 196 sujeitos, originados de 13 clínicas privadas de Audiologia e, embora tenha sofrido pequenas modificações, sua habilidade para quantificar a satisfação pelo acesso de seus componentes foi confirmada. É uma medida multidimensional designada para quantificar a satisfação indiretamente, a partir de seus elementos constituintes. O SADL fornece uma pontuação global e uma pontuação para cada uma de suas quatro subescalas.

Os itens são pontuados de tal forma que a satisfação é refletida pela alta pontuação. Uma pontuação é gerada para cada uma das quatro subescalas e a pontuação de cada subescala é computada a partir da média das respostas para suas questões. Para a pontuação ser considerada válida e utilizada na análise, todas as subescalas devem ser pontuadas. As quatro subescalas do SADL representaram quatro domínios diferentes que refletem os elementos constituintes da satisfação global. Então, a média das pontuações das subescalas, que são domínios da satisfação, resulta na pontuação de satisfação global.

A vantagem do SADL é que é um questionário viável para uso clínico, pois é relativamente curto, contendo apenas 15 questões, que acessam a natureza multidimensional da satisfação, permitindo a identificação de áreas de problemas ${ }^{(14)}$.

Neste estudo, foi utilizada a versão em Português Brasileiro do questionário SADL, disponibilizada pelos autores ${ }^{(15)}$.

Diante do exposto, o objetivo deste estudo foi adaptar o instrumento de avaliação do nível de satisfação de usuários de próteses auditivas, denominado SADL, versão em Português Brasileiro para administrar em usuários de AASIs dispensados pelo Sistema Único de Saúde (SUS).

\section{MÉTODOS}

Este estudo foi realizado na Divisão de Saúde Auditiva do Hospital de Reabilitação de Anomalias Craniofaciais (HRAC/ USP-Bauru), sendo este Centro credenciado como de alta complexidade pelo Sistema Único de Saúde (SUS).

O projeto foi aprovado pelo Comitê de Ética em Pesquisa da USP-Bauru, sob o processo $n^{\circ} 61 / 2008$, de 28 de maio de 2008. Os sujeitos que participaram do estudo assinaram o termo de Consentimento Livre e Esclarecido, junto à Carta de Informação ao Sujeito da Pesquisa.

\section{Sujeitos}

Participaram deste estudo 19 sujeitos, de ambos os gêneros, regularmente cadastrados no banco de dados do Sistema Único de Saúde. Os pacientes selecionados atenderam aos seguintes critérios: idade igual ou superior a 60 anos, no mínimo três semanas de uso do AASI, perda auditiva pós-lingual e que responderam todas as subescalas do SADL.

Foram excluídos do estudo os sujeitos que apresentavam grave limitação da capacidade de compreensão e expressão para responder ao questionário e os sujeitos que não concordaram em participar da pesquisa.

\section{Procedimentos}

Os sujeitos selecionados foram convidados a comparecer à Divisão de Saúde Auditiva do Hospital de Reabilitação de Anomalias Craniofaciais (HRAC-USP), para completarem o questionário. O questionário foi aplicado por uma avaliadora previamente treinada, a qual esclareceu as possíveis dúvidas, sem interferir de forma alguma nas respostas dos sujeitos.

Para assegurar a sensibilidade do questionário na avaliação do nível de satisfação dos sujeitos pesquisados foi aplicada também uma escala de satisfação com um item único, sugerida por Cox e Alexander ${ }^{(4)}$. A escala de satisfação de item único correspondeu a uma medida direta do nível de satisfação global dos sujeitos em relação ao seus AASIs. Esta escala envolveu uma única questão "Você está satisfeito com a sua prótese auditiva?", contemplando cinco diferentes alternativas de resposta, registradas desde "muito satisfeito" até "muito insatisfeito".

O questionário e a escala de satisfação de item único foram inicialmente aplicados de forma que o próprio paciente assinalava as respostas, com caneta e papel, como sugerido originalmente $^{(4)}$. Posteriormente, devido à dificuldade dos sujeitos em responder ao questionário, optou-se por a avaliadora realizar a leitura em voz alta e a anotação das respostas dos sujeitos pesquisados para os instrumentos utilizados, na forma de entrevista, possibilitando uma melhor compreensão das questões e alternativas de resposta.

Para facilitar o entendimento da escala de respostas, foi apresentada aos sujeitos uma escala com apoio visual, adaptando o instrumento para a realidade dos sujeitos pesquisados, já que os mesmos referiam não compreender a diferença entre níveis de resposta. Esta escala apresentou figuras representativas para os sete níveis de resposta, facilitando o entendimento de cada um (Figuras 1 e 2).

Ainda, os sujeitos foram questionados em relação a alguns itens adicionais propostos pelos autores ${ }^{(14)}$, referentes ao tempo de experiência e de uso diário dos AASIs. Foram registrados também alguns aspectos referentes aos aparelhos auditivos

Escala de resposta para questões 1, 3, 5, 6, 8, 9, 10, 11, 12, 14, 15

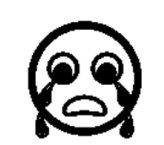

Nem um pouco
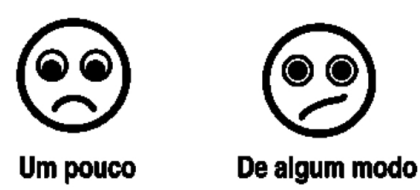

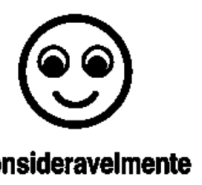

Consideravelmente
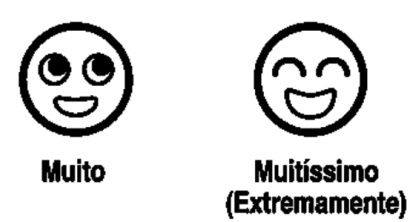

Figura 1. Escala de apoio visual adaptada à escala de respostas do SADL, para questões com pontuação não invertida Fonte: Adaptado de http://images.google.com.br/imgres?imgurl 


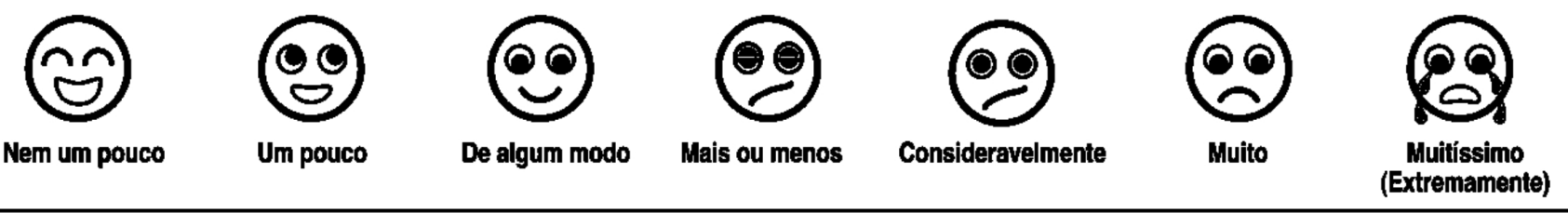

Figura 2. Escala de apoio visual adaptada à Escala de respostas do SADL, para questões com pontuação invertida Fonte: Adaptado de http://images.google.com.br/imgres?imgurl

e características audiológicas dos sujeitos, com o intuito de auxiliar na interpretação dos resultados e na determinação dos fatores associados à satisfação ou insatisfação dos usuários com seus AASIs.

\section{Pontuação}

Para responder às 14 questões de satisfação (a questão sobre custo foi omitida) foi utilizada uma escala de 7 pontos de igual intervalo, que correspondeu na escala categórica de "nem um pouco" a "muitíssimo" satisfeito. Para 11 questões, "muitíssimo" indicou total satisfação e foi pontuada com valor 7, enquanto "nem um pouco" indicou total insatisfação e foi pontuada com valor 1 . As outras quatro questões foram invertidas, onde "muitíssimo" indicou total insatisfação, sendo pontuada com valor 1 e "nem um pouco" indicou total satisfação, sendo pontuada com valor 7 (Quadro 1).

Quadro 1. Escala com os níveis de resposta e pontuação do SADL

\begin{tabular}{|lcc|}
\hline Registro & $\begin{array}{c}\text { Pontuação } \\
\text { não invertida }\end{array}$ & $\begin{array}{c}\text { Pontuação } \\
\text { invertida }\end{array}$ \\
\hline Nem um pouco & 1 & 1 \\
Um pouco & 2 & 6 \\
De algum modo & 3 & 5 \\
Mais ou menos & 4 & 4 \\
Consideravelmente & 5 & 3 \\
Muito & 6 & 2 \\
Muitíssimo & 7 & 1 \\
\hline
\end{tabular}

Com relação à escala de satisfação de um único item, a pontuação variou proporcionalmente ao nível de satisfação dos usuários, sendo registrada de 1 a 5 .

Foi atribuída uma pontuação de 1 a 5 para a escala de satisfação de item único: muito satisfeito $=5$, satisfeito $=4$, neutro $=3$, insatisfeito $=2$ e muito insatisfeito $=1$. Para verificar o relacionamento entre as duas escalas, foi atribuída a mesma pontuação para a escala SADL: muito satisfeito $=5$, satisfeito $=4$ e insatisfeito $=2$.

\section{Análise dos resultados}

Foi realizada a análise descritiva dos resultados, a fim de caracterizar as respostas dos sujeitos estudados, e uma avaliação da consistência interna do SADL por meio da pesquisa do Alpha de Crombach.
Para verificar o nível de satisfação dos sujeitos por meio das categorias "muito satisfeito", "satisfeito" e "insatisfeito", foi realizada a análise dos percentis. Foram calculados os percentis $20^{\circ}$ e $80^{\circ}$, por meio da seguinte fórmula:

$\mathrm{K}=$ percentil $(\mathrm{n}+1) / 100$

Nesta etapa, comparou-se os resultados dos percentis da amostra americana ${ }^{(4)}$ com os da amostra de sujeitos atendidos pelo SUS.

Posteriormente, foram comparados os níveis de satisfação relatados pelos sujeitos atendidos pelo SUS com a amostra americana $^{(4)}$ por meio do teste de Mann-Whitney.

Finalmente, foi realizada a análise da relação entre os resultados do SADL e os resultados da escala de satisfação de item único por meio de gráfico e valores médios.

O nível de significância adotado para os testes estatísticos foi de $5 \%$.

\section{RESULTADOS}

Participaram da pesquisa 19 sujeitos adaptados bilateralmente com AASIs dispensados pelo SUS, sendo $63 \%$ do gênero masculino e $37 \%$ do gênero feminino, com média de idade de 73 anos, média de tempo de uso dos AASIs de cinco meses.

A Tabela 1 descreve os resultados obtidos pelos sujeitos para a pontuação global e para as quatro subescalas do SADL: média, desvio-padrão, mediana, valores máximos e mínimos.

O coeficiente Alpha de Crombach geral para os sujeitos atendidos pelo SUS, calculado com todas as subescalas presentes, foi de 0,71 .

Tabela 1. Descrição da pontuação global e das quatro subescalas do SADL para os sujeitos pesquisados

\begin{tabular}{lcccccc}
\hline SADL & $\mathrm{n}$ & Média & $\mathrm{DP}$ & Mínimo & Mediana & Máximo \\
\hline Global & 19 & 5,2 & 0,9 & 3,0 & 5,3 & 6,6 \\
$\begin{array}{l}\text { Efeitos } \\
\text { positivos }\end{array}$ & 19 & 5,1 & 1,3 & 2,0 & 5,3 & 7,0 \\
$\begin{array}{l}\text { Serviço e } \\
\text { custo }\end{array}$ & 19 & 5,5 & 0,8 & 4,0 & 5,5 & 7,0 \\
$\begin{array}{l}\text { Fatores } \\
\text { negativos }\end{array}$ & 19 & 4,5 & 1,7 & 2,3 & 4,0 & 7,0 \\
$\begin{array}{l}\text { Imagem } \\
\text { pessoal }\end{array}$ & 19 & 5,9 & 0,9 & 4,0 & 6,0 & 7,0
\end{tabular}

Legenda: DP = desvio-padrão 
A Tabela 2 demonstra a comparação entre os valores de $20^{\circ}$ e $80^{\circ}$ percentis obtidos para os sujeitos atendidos pelo SUS e os obtidos em estudo americano ${ }^{(4)}$.

Tabela 2. Comparação entre os valores de $20^{\circ}$ e $80^{\circ}$ percentis obtidos pelos sujeitos atendidos pelo SUS e pela amostra americana ${ }^{(4)}$ na pontuação global e das quatro subescalas

\begin{tabular}{lcccc}
\hline \multicolumn{2}{l}{ Subscalas (SADL) } & $\begin{array}{c}\text { SUS } \\
\text { (HRAC-USP) }\end{array}$ & $\begin{array}{c}\text { EUA (Cox e } \\
\text { Alexander) }\end{array}$ & Diferença \\
\hline Global & $20^{\circ}$ & 4,8 & 4,2 & 0,6 \\
& $80^{\circ}$ & 6,1 & 5,9 & 0,2 \\
\hline Efeitos & $20^{\circ}$ & 4,2 & 3,8 & 0,4 \\
positivos & $80^{\circ}$ & 6,3 & 6,1 & 0,2 \\
\hline Serviço e & $20^{\circ}$ & 4,5 & 4,5 & 0 \\
custo & $80^{\circ}$ & 6,5 & 6,5 & 0 \\
\hline Fatores & $20^{\circ}$ & 2,7 & 2,3 & 0,4 \\
negativos & $80^{\circ}$ & 6,7 & 5,0 & 1,7 \\
\hline Imagem & $20^{\circ}$ & 5,0 & 5,0 & 0 \\
pessoal & $80^{\circ}$ & 6,7 & 6,7 & 0 \\
\hline
\end{tabular}

A Figura 3 descreve o nível de satisfação dos usuários de AASIs dispensados pelo SUS (HRAC-USP): Pontuação Global e das quatro Subescalas.

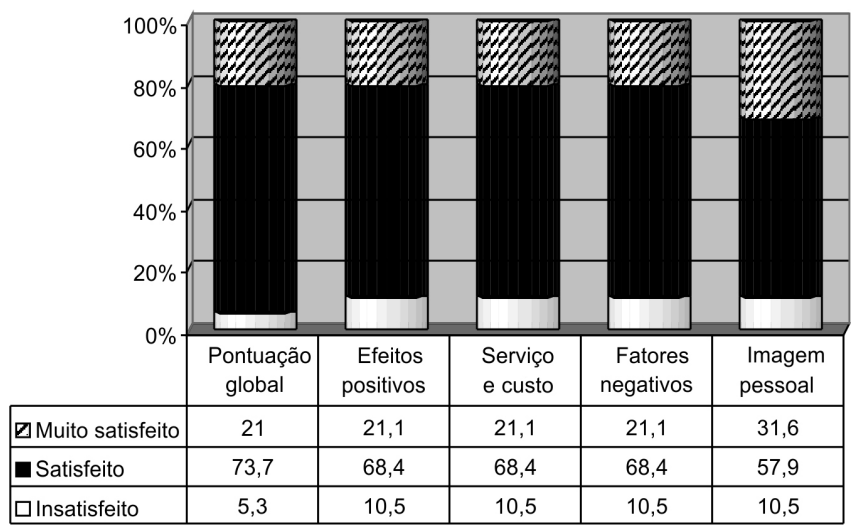

Figura 3. Nível de satisfação dos usuários de AASIs dispensados pelo SUS (HRAC-USP): pontuação global e de subescalas

Houve íntima relação entre a escala de satisfação de item único e a pontuação global do SADL. A maioria dos sujeitos apresentou a mesma pontuação nas duas escalas: os sujeitos que referiram estar "satisfeitos" na escala de satisfação de item único também estavam satisfeitos no SADL. O mesmo ocorreu nas outras classificações, como muito insatisfeito ou muito satisfeito (Figura 4).

O índice de correlação entre a pontuação global do SADL e a escala de satisfação de item único foi de 0,935 , indicando um índice de correlação alto e positivo.

\section{DISCUSSÃO}

Houve uma distribuição simétrica das pontuações do SADL relatadas pelos sujeitos atendidos pelo SUS. Os valores médios foram muito similares aos valores medianos, com exceção da subescala de Fatores Negativos (Tabela 1). A subescala de Fa-

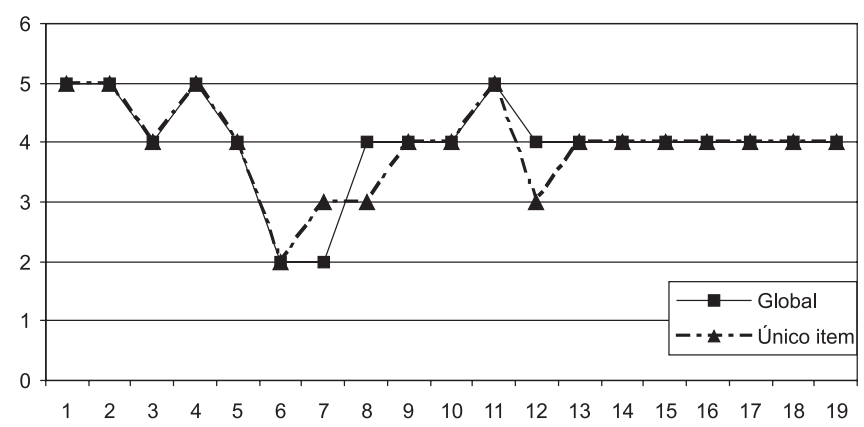

Figura 4. Comparação entre a pontuação global do SADL e a pontuação da Escala de Satisfação de Único Item

tores Negativos apresentou grande variabilidade de respostas, com pontuações voltadas para os maiores valores de satisfação.

O valor do Alpha de Crombach, de 0,71, demonstrou existir uma boa consistência interna do SADL. Alguns autores afirmam que valores de Alpha de Crombach acima de 0,7 são indicativos de boa consistência ${ }^{(16)}$.

Os resultados obtidos neste estudo demonstraram um alto nível de satisfação dos usuários de AASIs dispensados pelo SUS. Estes resultados foram equivalentes aos encontrados em estudo americano ${ }^{(4)}$ com sujeitos originados de um centro médico de veteranos, os quais não pagaram por seus aparelhos auditivos.

Para a pontuação global e de subescalas do SADL, os resultados médios obtidos pelos sujeitos atendidos pelo SUS comparados com a amostra americana, demonstraram não haver diferenças significativas entre eles. O teste de MannWhitney demonstrou um valor de $\mathrm{p}=0,2506(\mathrm{p}>0,05)$.

Em estudo para o desenvolvimento de normas provisórias para as quatro subescalas do SADL e para a pontuação global, foram propostas uma norma para sujeitos que pagaram por seus AASIs e outra, para sujeitos que receberam seus AASIs de terceiros ${ }^{(4)}$. Duas normas foram propostas: uma para sujeitos que pagaram por seus AASIs e outra para sujeitos que receberam seus AASIs de terceiros. Neste estudo foi realizada uma comparação dos resultados com os dados normativos referentes a sujeitos que receberam seus AASIs de terceiros, visto que este estudo ocorreu em serviço inserido no Sistema Único de Saúde, onde os aparelhos auditivos são dispensados sem qualquer custo ao paciente.

A variabilidade das pontuações obtidas para a subescala de Fatores Negativos também pôde ser observada quando foram comparados os percentis encontrados neste estudo (SUS) e os obtidos no estudo americano ${ }^{(4)}$. Para a pontuação global e das subescalas de Serviço e Custo, Imagem Pessoal e Efeitos Positivos foram encontrados percentis iguais ou muito semelhantes em ambos os estudos. Já a subescala de Fatores Negativos apresentou um valor de $80^{\circ}$ percentil, mais elevado neste estudo (Tabela 2).

Embora os sujeitos atendidos pelo SUS tenham demonstrado maior satisfação na subescala de Fatores Negativos, os resultados médios desta subescala não diferem dos encontrados no estudo anterior ${ }^{(4)}$.

Resultados semelhantes foram encontrados na validação do SADL ${ }^{(14)}$. Os autores encontraram resultados equivalentes aos obtidos na construção do instrumento, com exceção da 
subescala de Fatores Negativos. A subescala de Fatores Negativos apresentou uma pontuação muito superior à obtida na construção do SADL, demonstrando que houve maior satisfação dos sujeitos nesta subescala.

Outro estudo ${ }^{(17)}$ realizado no Brasil com usuários de AASIs fornecidos pelo Sistema de Saúde do Exército relatou resultados semelhantes aos obtidos nesta pesquisa. Os valores médios das subescalas foram similares aos descritos pelos autores e mantiveram a mesma configuração, tanto nos percentis quanto nas subescalas com maior ou menor satisfação. A subscala de Fatores Negativos demonstrou resultados indicando maior satisfação dos sujeitos do Sistema de Saúde do Exército, embora esta diferença não tenha se mostrado significativa.

A subescala de Fatores Negativos está relacionada a questões como o uso do telefone, microfonia e processamento do som na presença de ruído competitivo. Um fator que, provavelmente, contribuiu para uma maior satisfação dos sujeitos nesta subescala refere-se aos avanços tecnológicos apresentados pelos AASIs, como a transição do processamento linear para o processamento por compressão ${ }^{(14)}$, dentre outros.

Dois sujeitos apresentaram pontuações relativamente baixas na subescala de Efeitos Positivos, e um deles apresentou resposta relativamente baixa também na pontuação global. Estes valores corresponderam a pontos isolados na distribuição da amostra e promoveram um rebaixamento dos valores mínimos, observados na Tabela 1.

As respostas notavelmente baixas desses sujeitos para a subescala de Efeitos Positivos e pontuação global foram consideradas fatos isolados, e necessitam ser cuidadosamente investigadas quando ocorrem em ambiente clínico.

Verificou-se que um dos sujeitos (sujeito 6) apresentava muitas queixas com relação à manutenção do seu AASI. Alegou ter levado o mesmo para conserto devido ao "mau funcionamento" e ter demorado muito para recebê-la novamente. Este mesmo sujeito, também apresentou a menor pontuação na subescala de Serviço e Custo, a qual engloba questões relacionadas à manutenção do AASI.

Outra explicação também seria o fato dos dois sujeitos (sujeitos 6 e 7) serem os únicos nesse estudo a apresentarem perdas auditivas neurossensoriais de grau leve, em ambas as orelhas. Em estudo realizado na Austrália utilizando o SADL ${ }^{(18)}$, foi encontrada uma correlação pequena mas significativa entre o grau de perda auditiva e a pontuação global do SADL. Os sujeitos que apresentavam maiores perdas auditivas estavam mais satisfeitos com a amplificação que os sujeitos que apresentavam menores perdas auditivas. Em outro estudo sobre o benefício com o uso do AASI em populações de idosos ${ }^{(19)}$ foram encontrados benefícios não muito satisfatórios em sujeitos que apresentavam perdas auditivas de grau leve, quando comparados às demais perdas auditivas. Cabe ressaltar que a subescala de Efeitos Positivos (subescala onde os dois sujeitos apresentaram pontuações notavelmente baixas) está relacionada especificamente ao benefício com o uso do AASI, e consiste na subscala que tem maior influência na pontuação global do SADL, o que também poderia explicar as respostas baixas dos sujeitos em ambas as subescalas.
Por meio da pontuação global observou-se que os sujeitos, em média, encontravam-se satisfeitos com seus AASIs dispensados pelo SUS. O mesmo ocorreu para todas as subescalas do SADL, sendo que a subescala de Imagem Pessoal apresentou o maior número de pessoas "muito satisfeitas" (Figura 3).

Com relação à escala de satisfação de item único, observouse íntima relação desta com a pontuação global do SADL. Para as duas medidas de satisfação, a maioria dos sujeitos demonstrou estar "satisfeito" com seus AASIs. Ambas as escalas apresentaram uma correlação alta e significativa (Figura 4).

Foram adotadas algumas medidas auxiliares com o objetivo de minimizar as dificuldades geradas pela diferença cultural entre o grupo normativo e os sujeitos deste estudo, sendo sugerida a utilização das mesmas como medidas auxiliares à aplicação do SADL. Observou-se que a escala de apoio visual, elaborada como auxílio para a escala de respostas do SADL, foi outro fator determinante para a correta compreensão dos níveis de resposta pelos sujeitos, tendo em vista a diferença cultural existente entre os sujeitos deste estudo e os sujeitos do grupo normativo, bem como o baixo nível de escolaridade dos sujeitos deste estudo.

Ainda, uma questão mostrou-se de maior complexidade no momento de sua aplicação. A questão $\mathrm{n}^{\circ} 7$, referente ao feedback acústico, não foi bem compreendida pela maior parte dos sujeitos, sendo necessárias explicações adicionais. Mesmo com tal auxílio, quatro sujeitos deixaram de responder a referida questão.

A questão 7 também se mostrou problemática para os autores. Na construção original do instrumento( ${ }^{(4)}$ havia sido proposta da seguinte forma "Você está chateado por não conseguir mudar para o volume que deseja sem que seu aparelho apite?". Muitos AASIs não apresentavam controle de volume ou o mesmo não estava ativado, impossibilitando as respostas dos sujeitos.

$\mathrm{Na}$ validação do instrumento ${ }^{(14)}$, esta questão foi reescrita para "Você está chateado por não conseguir ter o volume que deseja sem que seu aparelho apite?", e sua utilização foi recomendada pelos autores, embora ainda tenha apresentado $3 \%$ de ausência de respostas.

Neste estudo, observou-se que, mesmo com a modificação, os usuários tenderam a interpretá-la como o item original, associando ao controle de volumes do aparelho. Todos os sujeitos que apresentaram ausência de resposta na questão 7 , apresentavam o controle de volume desativado.

Para adaptar culturalmente um instrumento é necessário aplica-lo em um pequeno grupo, que pode ser de 20,30 ou 40 sujeitos pertencentes ao público alvo ${ }^{(20)}$. Embora este estudo tenha promovido resultados confiáveis com a utilização do SADL versão em Português Brasileiro como instrumento para avaliar o nível de satisfação de usuários de AASIs dispensados pelo SUS, novas pesquisas são necessárias para complementar estes achados, englobando um maior número de sujeitos e também outros grupos de usuários de AASI.

\section{CONCLUSÃO}

Em média, os usuários de aparelhos auditivos dispensados pelo SUS estavam satisfeitos com seus AASIs. 
O questionário SADL, versão em Português Brasileiro, mostrou-se um instrumento eficaz para avaliar o nível de satisfação dos usuários de AASIs dispensados pelo SUS, avaliados neste estudo. Novas pesquisas são necessárias para complementar estes achados.

\section{AGRADECIMENTOS}

Agradecemos ao Conselho Nacional de Desenvolvimento Científico e Tecnológico (CNPQ), pelo apoio concedido para a realização dessa pesquisa, sob processo número 373104/2006-5.

\begin{abstract}
Purpose: To culturally adapt the Brazilian Portuguese version of the Satisfaction with Amplification in Daily Life (SADL) questionnaire to be administered in users of hearing aids dispensed by Brazil's Unified Health System (SUS). Methods: Participants were 19 subjects, users of hearing aids dispensed by SUS for at least three weeks, with ages 60 or older, who had post-lingual hearing loss. Sixty three percent of them were male and $37 \%$ were female, with mean age of 73 years and mean duration of hearing aids use of five months. Subjects answered the SADL questionnaire and a single-item Satisfaction Scale, besides a few additional items. The internal consistency analysis of the SADL was carried out, as well as the descriptive analysis of the results, in order to compare them to the normative data reported by the questionnaire authors. The relationship between SADL's global scores and the Satisfaction Scale were also investigated. Results: The results for the overall scores evidenced that subjects, in average, were satisfied with their hearing aids. The same was true for all subscales of the SADL, and the Personal Image subscale had the largest number of people very satisfied. The results were equivalent to those found by the questionnaire authors, and the SADL showed a good internal consistency (0.71). The single-item Satisfaction Scale was closely related to the global score of the SADL. For both satisfaction measures, most subjects demonstrated to be "satisfied" with their hearing aids, and there was a high significant correlation between both scales (0.935). Conclusion: In average, subjects were satisfied with their hearing aids dispensed by SUS. The Brazilian Portuguese version of the SADL proved to be an effective instrument to assess the level of satisfaction among the users of hearing aids dispensed by SUS evaluated in this study. Further research is necessary to complement these findings.
\end{abstract}

Keywords: Hearing aids; Hearing loss; Consumer satisfaction; Public health; Single health system

\title{
REFERÊNCIAS
}

1. Wilkerson D. Current issues in rehabilitation outcome measurement: implications for audiological rehabilitation. Ear Hear. 2000;21(4 Suppl):80S-88S

2. Kochkin S. Subjective measures of satisfaction and benefit: establishing norms. Semin Hear. 1997;18(1):37-48.

3. Almeida K, Russo ICP. Considerações sobre a seleção e adaptação de próteses auditivas para o idoso. In: Almeida K, Iorio MCM. Próteses auditivas: fundamentos teóricos e aplicações clínicas. São Paulo: Lovise; c1996. p. 177-90.

4. Cox RM, Alexander GC. Measuring Satisfaction with Amplification in Daily Life: the SADL scale. Ear Hear. 1999;20(4):306-20.

5. Abrams HB, McArdle R, Chisolm TH. From outcomes to evidence: establishing best practices for audiologists. Semin Hear. 2005;26(3):15769.

6. Cox RM, Alexander GC, Gray GA. Hearing aid patients in private practice and public health (Veterans Affairs) clinics: are they different? Ear Hear. 2005;26(6):513-28.

7. Brasil. Ministério da Saude. Portaria GM/MS nº 2.073 de 28 de setembro de 2004. Institui a Política Nacional de Atenção à Saúde Auditiva. Diário Oficial da União. 29 set 2004; Seção 1:34.

8. Almeida SM, Manzoni CR, Tedesco MR, Leite SH. A implantação da política de saúde auditiva no município de São Paulo. In: II Seminário Científico "Políticas Públicas em Saúde Auditiva”. Anais. São Paulo; 2008.

9. Cook JA, Hawkins DB. Outcome measurement for patients receiving hearing aid services. Laryngoscope. 2007;117(4):610-3.

10. Armigliato MA, Prado DG, Melo TM, Martinez MA, Lopes AC, Amantini RC, Bevilacqua MC. Avaliação de serviços de saúde auditiva sob a perspectiva do usuário: proposta de instrumento. Rev Soc Bras Fonoaudiol. 2010;15(1):32-9.

11. Cox RM, Alexander GC. Expectations about hearing aids and their relationship to fitting outcome. J Am Acad Audiol. 2000;11(7):368-82; quiz 407.

12. Cox RM, Alexander GC. Maturation of hearing aid benefit: objective and subjective measurements. Ear Hear. 1992;13(3):131-41.

13. McLeod B, Upfold L, Broadbent C. An investigation of the applicability of the inventory, satisfaction with amplification in daily life, at 2 weeks post hearing aid fitting. Ear Hear. 2001; 22(4):342-7.

14. Cox RM, Alexander GC. Validation of the SADL questionnaire. Ear Hear. 2001;22(2):151-60.

15. Cox RM, Alexander GC. Satisfação com o aparelho auditivo em sua vida diária. [cited 2008 Maio 20]. Disponível em: http://www.memphis.edu/ ausp/harl/sadl.htm

16. Streiner DL, Norman GR. Health measurement scales: a practical guide to their development and use. 3rd ed. Oxford: Oxford University Press; 2005.

17. Veiga LR, Merlo ARC, Mengue SS. Satisfação com a prótese auditiva na vida diária em usuários do Sistema de Saúde do Exército. Rev Bras Otorrinolaringol. 2005;71(1):67-73.

18. Uriarte M, Denzin L, Dunstan A, Sellars J, Hickson L. Measuring hearing aid outcomes using the Satisfaction with Amplification in Daily Life (SADL) questionnaire: Australian data. J Am Acad Audiol. 2005;16(6):383-402.

19. Costa MHP, Sampaio ALL, Oliveira CACP. Avaliação do benefício da prótese auditiva digital e da percepção da desvantagem auditiva ou "handicap" em idosos não institucionalizados. Arq Int Otorrinolaringol. 2007;11(2):159-68.

20. Beaton D, Bombardier C, Guillemin F, Ferraz MB. Recommendations for the Cross-Cultural Adaptation of Health Status Measures. American Academy of Orthopaedic Surgeons and Institute for Work \& Health. Revised March 2002. Available from:http://www.dash.iwh.on.ca/assets/ images/pdfs/xculture2002.pdf 
Anexo 1. SADL - versão em Português Brasileiro(15)

\section{Satisfação com o aparelho auditivo em sua vida diária}

Nome

Data de nascimento _______ data ___ _ _

Instruções

Estão listadas abaixo, perguntas sobre o seu aparelho auditivo. Favor circular a letra correspondente a melhor resposta para você, para cada pergunta. A lista de palavras à direita Ihe oferece o significado de cada letra.
A Nada
D Mediamente
F Muito
B Um pouco
E Consideravelmente
G Muitíssimo

C De alguma forma

Lembre-se que suas respostas devem mostrar suas opiniões gerais em relação ao aparelho auditivo que você está usando agora ou aquele que utilizou mais recentemente.

\begin{tabular}{|c|c|}
\hline $\begin{array}{l}\text { 1. Seus aparelhos auditivos Ihe ajudam a entender o que as pessoas que conversam mais frequentemente com } \\
\text { você falam, quando comparado sem o uso dos aparelhos? }\end{array}$ & A B C DEFG \\
\hline $\begin{array}{l}\text { 2. Você fica frustrado quando o seu aparelho capta sons que não permitem que você ouça os sons que gostaria } \\
\text { de ouvir? }\end{array}$ & A B C D E F G \\
\hline 3. Você está convencido de que adquirir os seu aparelhos foi sua melhor opção? & A B C DEFG \\
\hline $\begin{array}{l}\text { 4. Você acha que as pessoas percebem mais a sua perda aditiva quando você está usando o seu aparelho } \\
\text { auditivo? }\end{array}$ & A B C D E F G \\
\hline $\begin{array}{l}\text { 5. Os seus aparelhos reduzem o número de vezes que você tem que pedir para as pessoas repetirem o que } \\
\text { disseram? }\end{array}$ & A B C D E F G \\
\hline 6. Você acha que o seu aparelho compensa seu problema? & A B C D E F G \\
\hline 7. Você está chateado por não conseguir ter o volume que deseja sem que o aparelho apite? & A B C D E F G \\
\hline 8. O quanto você está satisfeito com a aparência de seus aparelhos? & A B C DEFG \\
\hline 9. Usar o aparelho melhora a sua autoconfiança? & A B C DEFG \\
\hline 10. Quão natural é o som que recebe de seu aparelho? & A B C D EFG \\
\hline $\begin{array}{l}\text { 11. O quanto seus aparelhos ajudam ao falar em telefones que não tenham amplificadores de volume? (Se } \\
\text { você escuta bem ao telefone sem os aparelhos selecione aqui } \bigcirc \text { ) }\end{array}$ & A B C D E F G \\
\hline 12. Quão competente era a pessoa que lhe forneceu os aparelhos? & A B C DEFG \\
\hline 13. Você acha que usar o aparelho faz você se sentir menos capaz? & A B C DEFG \\
\hline 14. O custo do seu aparelho Ihe parece razoável? & A B C D E F G \\
\hline $\begin{array}{l}\text { 15. Você está satisfeito com a qualidade do seu aparelho (com relação ao número de vezes que ele precisou } \\
\text { de reparo)? }\end{array}$ & A B C D E F G \\
\hline
\end{tabular}

